\title{
Integration and Spike Initiation in Neuronal Terminals
}

\author{
Lolin T. Wang-Bennett and Raymon M. Glantz \\ Department of Biology, Rice University, Houston, Texas 77005
}

\begin{abstract}
Axoaxonal synapses impinge on the terminals of neurons and are thought to play a crucial role in the regulation of synaptic transmission. In the crayfish brain, the terminals of several identified interneurons exhibit both EPSPs and IPSPs in response to cephalic sensory stimulation. The terminal arbors extend into primary sensory neuropils near the terminals of primary afferent axons of the antennae and statocysts. The EPSPs arise monosynaptically from primary afferent stimulation and can elicit action potentials. Bidirectional conduction is routinely observed. Terminal IPSPs are transmitted via a polysynaptic pathway. Furthermore, two morphological classes of synaptic profiles impinge on the presynaptic terminals. These results indicate that the neuron terminal can function as a second, independent site of synaptic integration.
\end{abstract}

The neuron doctrine (Shepherd, 1979) is based on the compartmentalization of cell functions and the dynamic polarization of the nerve cell structure, i.e., integration on dendrites, action potential propagation in the axon, and transmitter release at the terminal. In recent years the neuron concept has been expanded to include dendrodendritic and axoaxonal synapses (Shepherd, 1979). In general, axoaxonal synapses are thought to modulate transmitter release during presynaptic inhibition (Atwood, 1976; Dudel, 1965; Dudel and Kuffer, 1961) and heterosynaptic facilitation (Kandel and Tauc, 1965; Klein and Kandel, 1980).

Presynaptic inhibition is a widespread phenomenon in crayfish nerve tissue. It is observed at the neuromuscular junction (Atwood, 1976; Dudel, 1965; Dudel and Kuffler, 1961) and at the terminals of primary afferents (Kennedy et al., 1974; Krasne and Bryan, 1973). More recently, these observations have been extended to synapses between interneurons (Fricke et al., 1982; Glantz et al., 1985). With a few exceptions (Fuchs and Getting, 1980; Kawai and Niwa, 1978; Kirk and Wine, 1984; Koketsu, 1956), both presynaptic inhibition and heterosynaptic facilitation are assessed by measurements in the postsynaptic cell, which indirectly reflect the amount of transmitter released by the presynaptic neuron. The synaptic physiology of the nerve terminal in the CNS is largely unexplored. In this study, the terminals of identified interneurons were directly impaled for physiological examination and dye-filled for subsequent histology. The terminals revealed multiple sources of synaptic input, the occurrence of EPSPs and IPSPs and two morphological classes of synaptic boutons impinging on the same terminal arbor.

\section{Materials and Methods}

Adult crayfish (Procambarus clarkii) measuring 8-12 cm were used in this experiment. The supraesophageal ganglion (brain) was exposed and electrophysiological recordings were made in vivo. The dissection and recording methods have been reported elsewhere (Glantz et al., 1981,

\footnotetext{
Received Aug. 1, 1985; revised Nov. 18, 1985; accepted Dec. 18, 1985

This research was supported by NSF Grant BNS 83-02701 to R.M.G. and NIH Postdoctoral Fellowship 5T32 EY07024 to L.T.W-B.

Correspondence should be addressed to R. M. Glantz, Department of Biology, Rice University, P.O. Box 1892, Houston, TX 77251 .
}

Copyright (C) 1986 Society for Neuroscience 0270-6474/86/061726-07\$02.00/0
1985). The focus of this study is the terminals of ascending interneurons These neurons originate in thoracic and abdominal ganglia. Their axons run through the ventral nerve cord and circumesophageal connectives, and they terminate in the brain.

The axon terminals were penetrated in the tritocerebrum of the brain with beveled micropipettes (ca. $100 \mathrm{M} \Omega$ ) backfilled with $2 \mathrm{M} \mathrm{K}^{+}$-acetate, a mixture of HRP (2.0\%) and $\mathrm{KCl}(1.5 \%)$, or a $3.0 \%$ solution of Lucifer yellow in distilled water (Stewart, 1978). Platinum hook electrodes were placed on each circumesophageal connective for recording and stimulation. Signals were stored on an FM tape recorder and analyzed offline with the aid of a digital oscilloscope (Norland Prowler).

The ascending interneurons were identified by four criteria: (1) rostral direction of spike propagation between a connective electrode and the brain (Fig. 1b); (2) absence of a prepotential at the base of the spike (Fig. 1b); (3) an extensive, multisegmental sensory receptive field caudal to the cephalic carapace; and (4) the presence of a terminal arbor and absence of a cell body in the brain (Fig. 1a). The caudal receptive field was measured manually with brushes for tactile stimulation and forceps for bending joints. Synaptic potentials were elicited by mechanical pulses (from a speaker coil) to the antennae or statocysts or by electrical shocks to the associated afferent root branches. The conduction delay of the afferent volley was about 1.5 msec (Glantz, 1978).

Bidirectional conduction was assessed off-line with the aid of a twochannel digital oscilloscope. The oscilloscope was triggered on the rising phase of the intracellularly recorded action potential, and the time of occurrence of the extracellularly recorded axonal spike was displayed on the second channel (Fig. $1, b, c)$.

Following the physiological identification of the interneuron, Lucifer yellow or HRP was iontophoresed into the cell. The procedures for Lucifer yellow and HRP injection and fixation followed those described previously (Wang-Bennett and Glantz, 1985). HRP-labeled specimens were fixed in $3 \%$ glutaraldehyde in $0.1 \mathrm{~m}$ phosphate buffer. HRP reaction product was visualized in the EM by using the chromogen $3,3^{\prime}-\mathrm{di}$ aminobenzidine tetrahydrochloride.

\section{Results}

Multisegmental, ascending interneurons arise in the abdominal and thoracic ganglia of the crayfish nervous system (Wiersma and Bush, 1963; Wiersma and Hughes, 1961) and terminate in the brain. At the light-microscopic level, the terminal of an ascending cell consists of a tapering axonal process (5-15 $\mu \mathrm{m}$ in diameter) that projects numerous varicose branches into the neuropil (Fig. la). The branches generally terminate in the paraolfactory lobe of the deutocerebrum or the antennal lobe of the tritocerebrum. The paraolfactory and antennal lobes contain the terminals of the statocyst and antennal nerves, respectively. These two neuropilar regions thus provide a plausible substrate for synaptic interactions between the cephalic sensory pathways and the ascending interneuronal terminal.

Synaptic potentials elicited by afferent root shock (to antennae or statocyst) were in some instances diphasic (Fig. $2 a$ ), with a short-latency depolarizing component (2-3 msec) and a longer latency hyperpolarizing component $(5-20 \mathrm{msec})$. More commonly, however, both early and late events were depolarizing at resting potential. Membrane polarization demonstrates that the late event is an IPSP (Fig. 2b). Hyperpolarization of the membrane produced large increases in the size of the late PSP, and depolarization diminished and reversed the late response. 

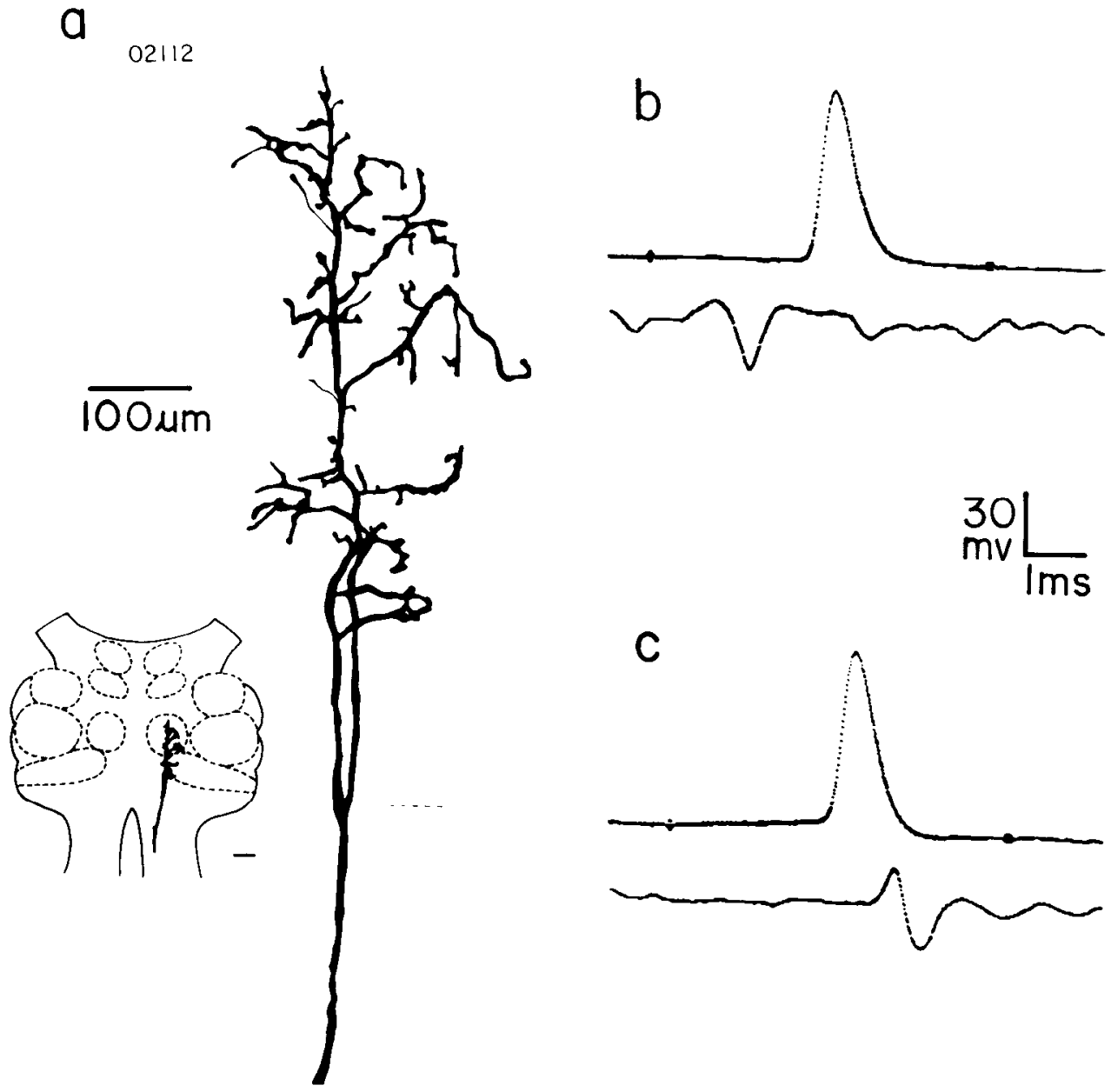

Figure 1. Structure and physiology of two-way conducting cells. $a$, Drawing of a terminal of an ascending interneuron in craytish brain. Dashed line indicates where axon (below line) enters the brain. Insert, (lower left), Terminal arbor in the tritocerebrum and paraolfactory lobe. Insert scale, $200 \mu \mathrm{m}$. $b$, Intracellular action potential in nerve terminal (top trace) preceded by spike in circumesophageal connective (lower trace), indicating orthodromic conduction. Conduction delay (measured from the negative trough of the extracellular spike to the midpoint of the rising phase of the intracellular spike), $1.15 \mathrm{msec} . c$, Intracellular action potential (top trace) elicited by tactile stimuli to the antennae precedes spike in circumesophageal connective (lower trace), indicating antidromic conduction. Conduction delay, 1.15 msec. For $b$ and $c$, signal average of 16 traces. Scale based on intracellular recording.

The reversal potential of the late PSP was $11.5 \pm 4.9 \mathrm{mV}(n=$ 9) above rest. The late PSP reversed below the spike threshold and was never observed to elicit an impulse. Qualitatively similar results in 40 preparations established that the late event was an IPSP. The IPSP was associated with a large increase in terminal input conductance, and orthodromic spikes were attenuated if superposed on the IPSP. All of these features are consistent with a role in presynaptic inhibition (Glantz et al., 1985).

PSPs in a single terminal could be elicited by stimuli applied to from two to four afferent pathways (Fig. 3) of the brain. Furthermore, the IPSPs were invariably compound events, with numerous subcomponents arriving between 5 and $150 \mathrm{msec}$ poststimulus. These observations imply that the terminal arbor is a target for convergent inputs.

In many preparations, the early PSP could give rise to one or more action potentials without additional membrane polarization (Fig. 3). On the basis of these results we conclude that the early event is an EPSP. Its brief, fixed latency $(2-3 \mathrm{msec})$ and resistance to fatigue suggest that it arises by a direct synaptic connection from the cephalic primary afferents (Glantz, 1978). The above results imply that the cells are capable of bidirectional conduction. To test this directly, we elicited spike trains by applying manual tactile stimuli exclusively to caudal structures in one epoch and to cephalic structures in another. With caudal (i.e., ipsilateral walking leg) stimulation, every action potential was preceded by an extracellular connective spike by $1.1 \mathrm{msec}$ (interelectrode distance, $4.0 \mathrm{~mm}$; Fig. $1 \mathrm{~b}$ ), and there was never a sign of a phase-locked spike following the terminal action potential. Thus, caudal stimuli promote ascending conduction. During epochs of cephalic stimulation, the terminal spike always preceded the connective spike (Fig. 1c), and there was never a sign of a phase-locked connective spike preceding the terminal action potential. Thus, cephalic stimuli promote descending action potentials.

Although there is extensive overlap in the receptive fields of the early and late events, the two fields are not identical. In one ascending interneuron, the response to stimulation of the antennal nerve (Fig. $3 a$ ) consisted of EPSPs and action potentials. Both the initial doublet and the delayed spike were followed by a postspike hyperpolarization of about $2.0 \mathrm{mV}$. A shock to the antennule nerve elicited a similar spike doublet, but the postspike hyperpolarization was $5.0 \mathrm{mV}$ (Fig. $3 b$ ). We infer that the larger hyperpolarization shown in Figure $3 b$ was due to a delayed hyperpolarizing IPSP elicited by the antennule nerve shock. The early and late events can also be separated by threshold. In a different preparation, increasing the shock intensity to the statocyst root first elicited the late event (Fig. $3 c$, lower trace). The EPSP required a higher stimulus intensity (Fig. $3 c$, upper trace).

The HRP-injected ascending interneurons that exhibited terminal PSPs were first visualized in thick sections with the LM and subsequently thin-sectioned for electron microscopy. The synaptic profiles observed in thin sections were identified by the usual criteria, including a distinct cluster of vesicles surrounding an electron-dense tuft in the presynaptic ending (King, 1976). The HRP-injected neurons exhibited high densities of small, round vesicles at numerous loci in the terminal arbor (Fig. 4a), 

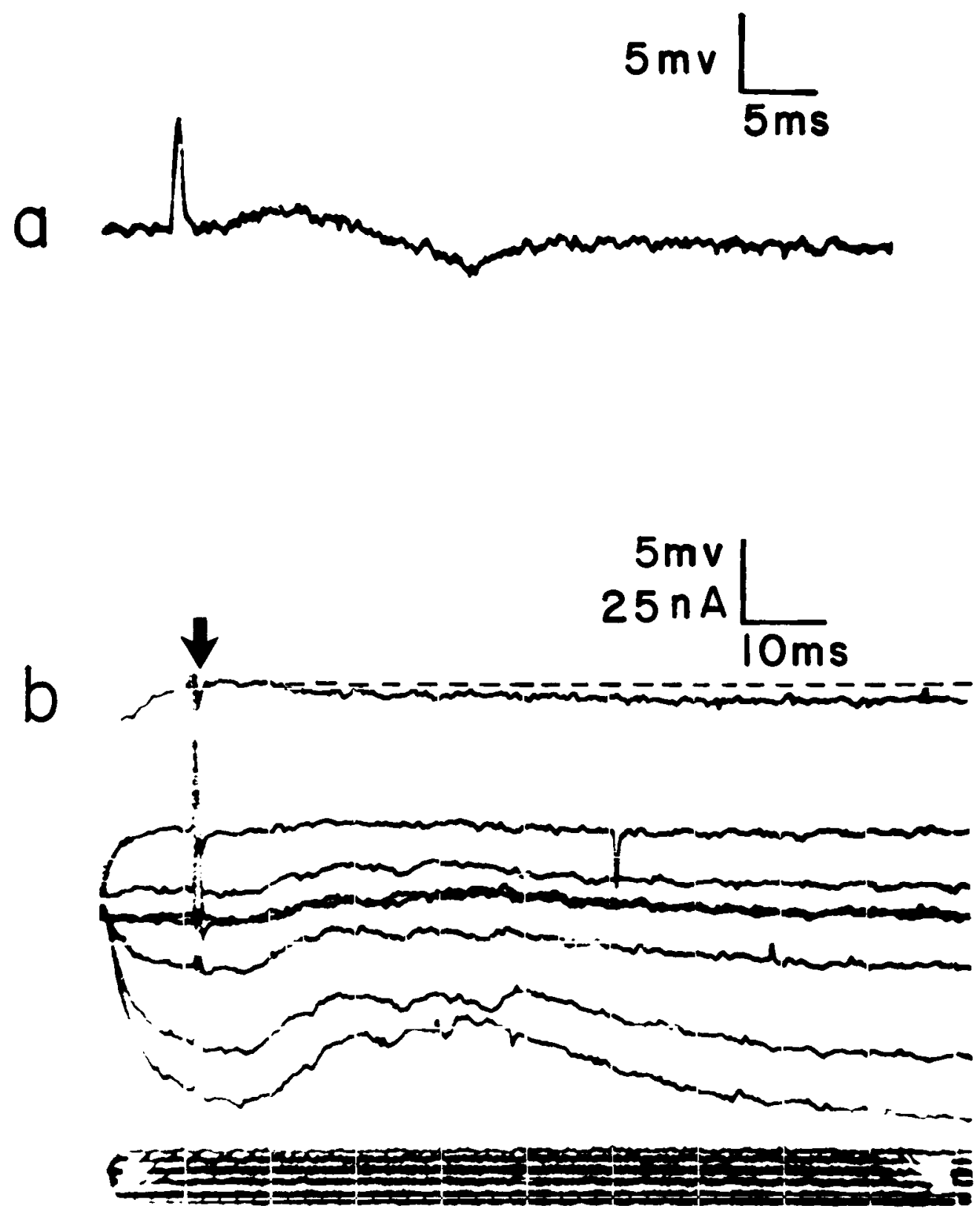

Figure 2. Synaptic physiology of neuronal terminals. $a$, Diphasic PSP in terminal elicited by a shock to the ipsilateral antennal nerve. $b$, Shock to ipsilateral antennal nerve elicits a delayed PSP (latency, $6 \mathrm{msec}$ ) in nerve terminal. Hyperpolarization increases and depolarization decreases and reverses the PSP. A depolarization of $14 \mathrm{mV}$ (uppermost trace) inverts the PSP. Dashed line, A depolarization of $14 \mathrm{mV}$.

and presynaptic electron-dense tufts (Fig. $4 a$, adjacent to open arrow) could be seen above the background of the HRP reaction product.

In aldehyde-fixed vertebrate central nerve tissue and crayfish peripheral nerve tissue there is evidence to suggest that excitatory and inhibitory endings can be distinguished by the shape of the vesicles (Atwood et al., 1972; Nakajima et al., 1973; Uchizono, 1965, 1967). Excitatory terminals exhibit round vesicles, and inhibitory endings have flattened or oval vesicles. Commensurate with this distinction, we found that the HRP-filled ascending cells contained round vesicles (Fig. $4 a$ ), and that their synaptic influence in the brain was excitatory (Fig. 5; also see Glantz, 1978; Wiersma and Mill, 1965). The micrographs in Figure $4, b-d$, were derived from the same cell that yielded the short-latency EPSP and long-latency IPSP in Figure 3c. Synaptic profiles were found to impinge at numerous sites on the terminal arborization. Some profiles contained only round vesicles (Fig. $4 a$ ) or predominantly round vesicles (Fig. $4 b$, Ex), while others exhibited a preponderance of flattened vesicles (Fig. 4, $c$ and $d$, In). The vesicles were measured to determine whether the eccentricities gave rise to a dichotomy of synaptic profiles or if they merely varied along a continuum. The long and short axes of all vesicles in 36 synaptic profiles were measured, and the mean long/short $(\mathrm{L} / \mathrm{S})$ axis ratio was determined for each profile. The mean L/S ratios fell into two well-defined groups (Fig. 6). Twenty-one of the 36 profiles contained a preponderance of round vesicles. These profiles exhibited mean $\mathrm{L} / \mathrm{S}$ ratios in the range of 1.05-1.30 and a population mean of $1.17 \pm 0.05$ (SD). The remaining 15 profiles (flat) exhibited mean $\mathrm{L} / \mathrm{S}$ ratios of $1.71-2.40$ and a population mean of $1.94 \pm 0.20$ (SD). The difference was highly significant $(p<0.005)$. This result supports the contention that there are two classes of synaptic pro- 

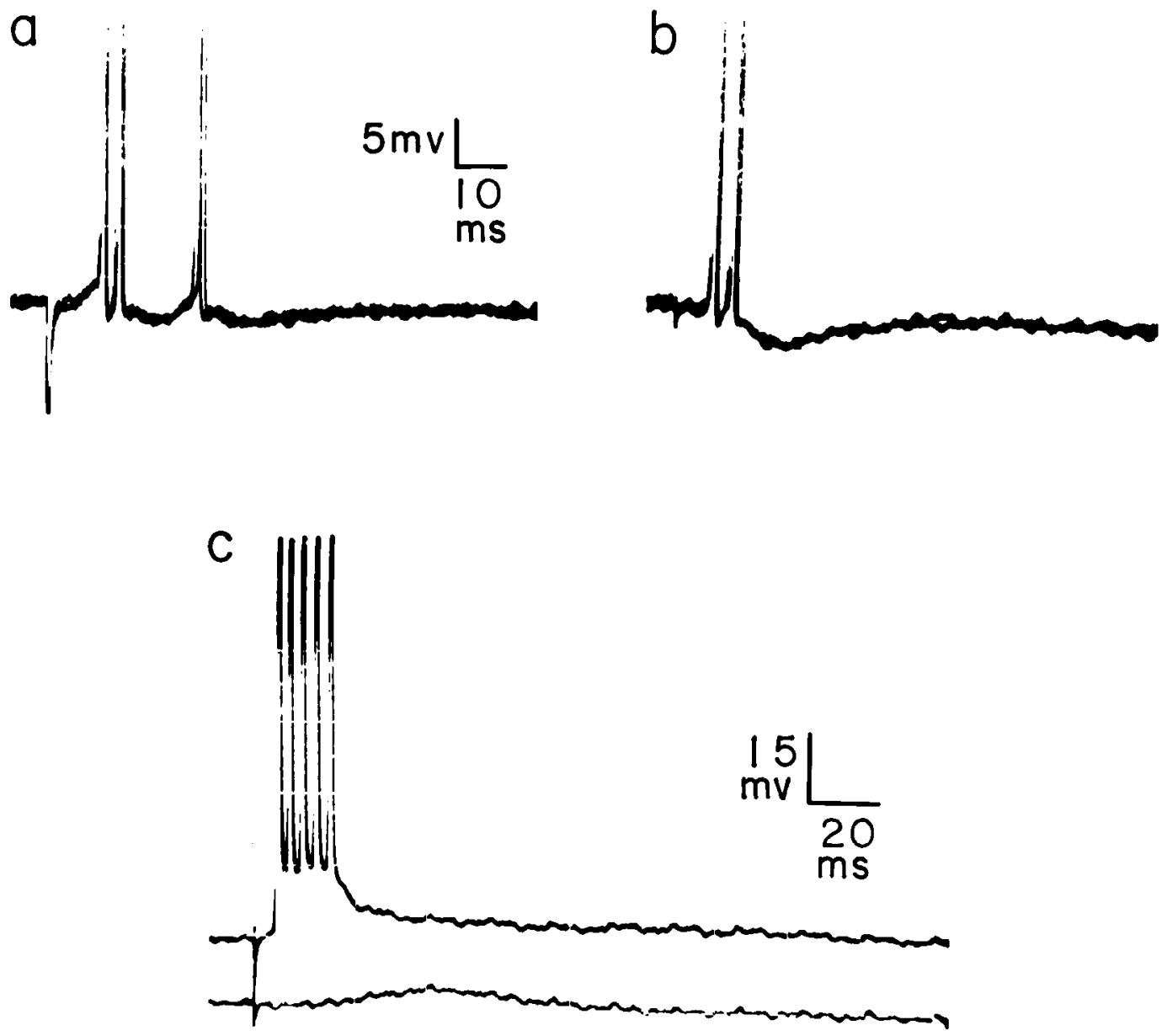

Figure 3. Afferent root shocks elicit EPSPs and spikes in ascending interneuronal terminals. $a$, Shock to antennal nerve elicits EPSPs and three action potentials (superposition of three traces). $b$, Shock to the antennule nerve also elicits both EPSPs and action potentials. The hyperpolarization following the doublet in $b$ is $5.0 \mathrm{mV}$, while in $a$ it is only $2.0 \mathrm{mV}$. $c$, In a different preparation, a low-intensity shock to the antennule nerve gives rise to late PSP (lower trace). Stronger shock elicits an early PSP and a burst of action potentials (upper trace).

files. We propose that the round and flat vesicle synaptic profiles are associated with the monosynaptic EPSP and polysynaptic IPSP, respectively.

\section{Discussion}

In two recent studies (Glantz et al., 1985; Wang-Bennett and Glantz, 1985), we proposed that IPSPs in the terminals of ascending interneurons are associated with presynaptic inhibition of the synapses between ascending neurons and the interneurons of the brain. The terminal EPSP has several possible functional roles: (1) graded transmitter release (Blight and Llinás, 1980; Burrows and Siegler, 1978) by ascending interneurons, (2) heterosynaptic facilitation, and (3) antidromic spike activation.

The simplest hypothesis to test, from a technical standpoint, is that the terminal EPSP supports antidromic spike activation. In most of the cells examined in this study, stimuli applied to cephalic sense organs or nerve roots elicited one or a train of action potentials (Figs. $1 c, 3$ ). Afferent root shocks and mechanical pulses to the statocyst can elicit spikes with 3-4 msec latencies. These latencies are significantly shorter than the minimum reflex loop times for activation via synapses in other ganglia. Additionally, the voltage threshold for the action potential in the terminal segment of the ascending cell axon is similar to that in the rest of the axon (Glantz et al., 1985), and the current threshold is somewhat smaller (owing to the higher input resistance of the terminal as compared to the axon in the connectives). Thus, a local mechanism for spike initiation is present. Finally, bidirectional axonal conduction is directly demonstrable (Fig. 1, $b, c$ ) with physiological stimuli. The latter result is in accord with earlier studies (Wiersma and Bush, 1963; Wiersma and Hughes, 1961; Wiersma and Mill, 1965) which revealed several bidirectionally conducting intersegmental interneurons in crayfish nerve cord. It is interesting to note in this regard that, among our sample of physiologically identified bidirectional cells, we discovered one neuron with the morphology of a descending interneuron, i.e., soma and dendrites in the brain.

The ascending neuronal terminals thus exhibit synaptic input from multiple sources, excitatory as well as inhibitory synapses, and a local spike-initiating zone. These are all hallmarks of an integrative mechanism commonly associated with the "input end" of the cell. Since dendrodendritic synapses are rather common in arthropod nervous systems (Glantz, 1978; Graubard et al., 1980; King, 1976); it is likely that some of these neurons can function in intercellular communication equally well in both directions. So long as both ends are not activated at the same time, a bidirectional cell can represent an economical way of coordinating activities between different segments of the nervous system or for the communication of sensory information in pathways not concerned with the location of the stimulus. 

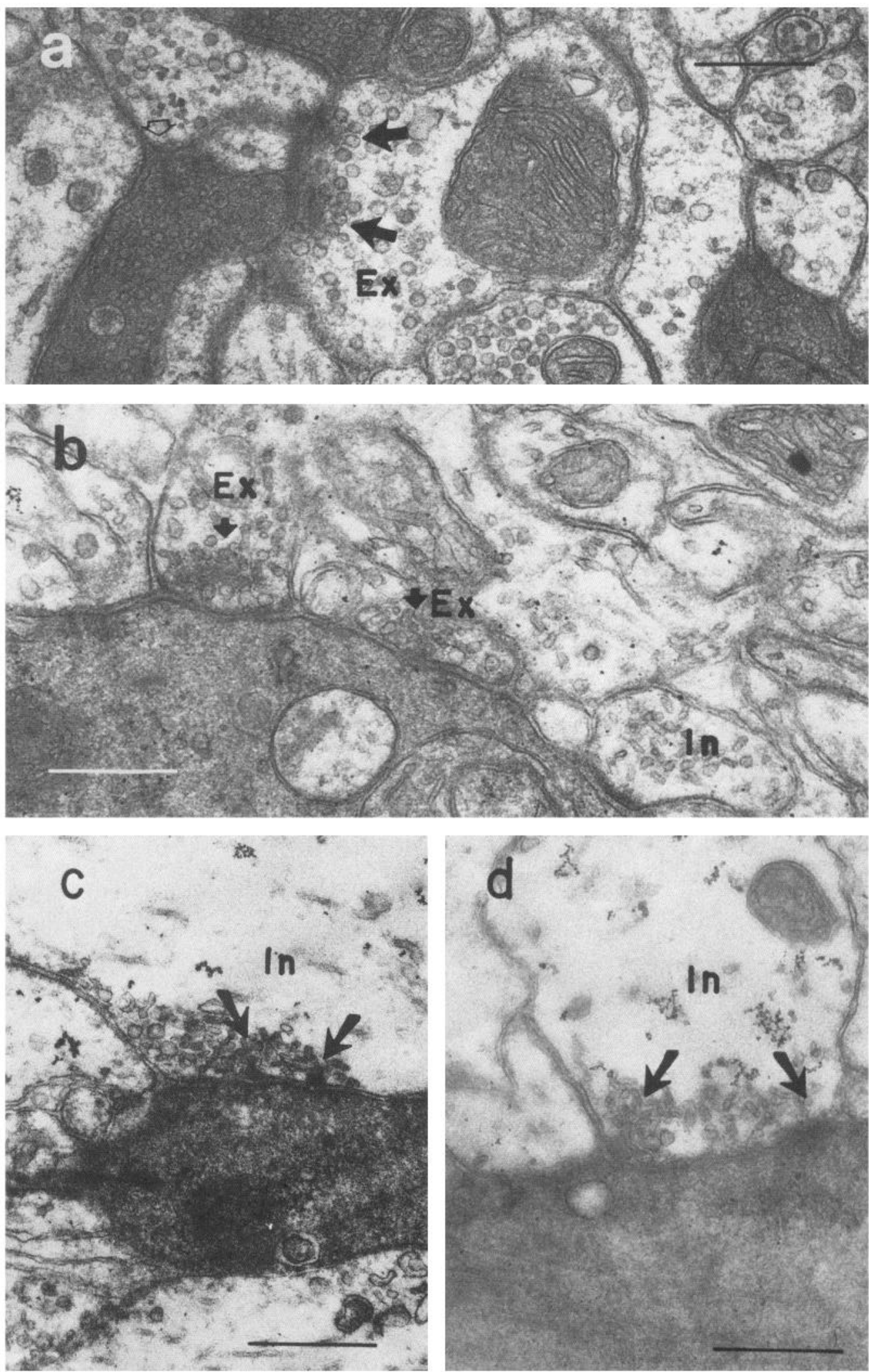

Figure 4. Two classes of synaptic profiles impinge on the terminal of an HRP-filled ascending interneuron in the brain. $a$, Excitatory (Ex) synaptic profile with round vesicles (arrows) and electron-dense tuft opposite HRP-filled cell. Open arrow is opposite electron-dense tuft in HRP-filled neuron. $b-d$, From the same cell that yielded results of Figure $3 c$. $b$, Two excitatory profiles synapse on the interneuron, but the inhibitory profile (In) does not. $c$ and $d$, Inhibitory profiles synapse on different branches of terminal arbor. Scale, $0.5 \mu$ m. Specimens were fixed in glutaraldehyde and then osmium tetroxide; thin sections were stained with lead citrate and uranyl acetate. 


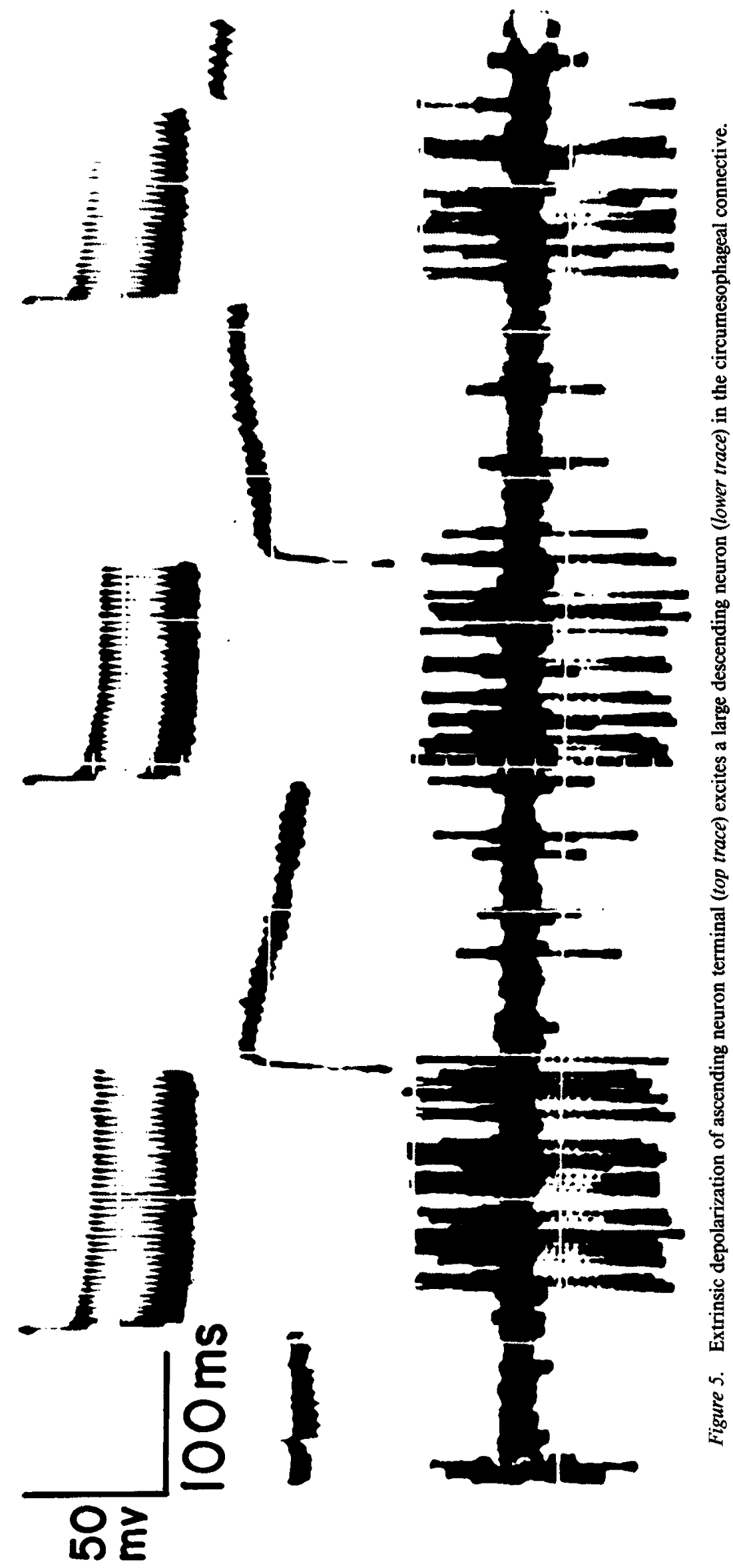




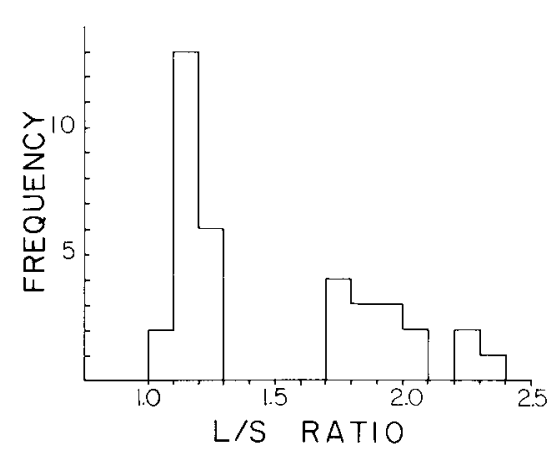

Figure 6. Bimodal distribution of synaptic profiles. The long and short axes of the vesicles in 36 synaptic profiles were measured, and the ratio of the long : short axes $(\mathrm{L} / \mathrm{S})$ determined for each vesicle. The mean $\mathrm{L} / \mathrm{S}$ ratio was determined for each profile, and the 36 mean ratios plotted in the frequency histogram. For the 21 round vesicle profiles, the mean $\mathrm{L} / \mathrm{S}$ ratio was $1.17 \pm 0.05$ (SD). For the 15 flat vesicle profiles, the mean ratio was $1.94 \pm 0.20(\mathrm{SD})$. The difference is highly significant $(p<$ $0.005)$.

The neurons sampled in this study have the features of multisegmental sensory interneurons, as originally described by Wiersma and Hughes (1961) and Wiersma and Bush (1963).

The proximity of the observed axoaxonal synapses to the ascending cell's release sites suggests that the terminal EPSP might function in heterosynaptic facilitation or graded synaptic transmission. Preliminary tests for graded transmission used an experimental protocol similar to that employed for the result shown in Figure 5, but with subthreshold current pulses injected into the terminal. The results were inconclusive. The possible role of the terminal EPSP in heterosynaptic facilitation has not been examined.

\section{References}

Atwood, H. L. (1976) Organization and synaptic physiology of crustacean neuromuscular systems. Prog. Neurobiol. 7: 291-391.

Atwood, H. L., F. Lang, and W. A. Morin (1972) Synaptic vesicles: Sclective depletion in crayfish excitatory and inhibitory axons. Science 176: 1353-1355.

Blight, A. R., and R. Llinás (1980) The non-impulsive stretch-receptor complex of the crab: A study of depolarization-release coupling at a tonic sensorimotor synapse. Phil. Trans. R. Soc. [Biol.] 290: 219276.

Burrows, M., and M. V.S. Siegler (1978) Graded synaptic transmission between local interneurones and motor neurones in the metathoracic ganglion of the locust. J. Physiol. (Lond.) 285: 231-255.

Dudel, J. (1965) The mechanism of presynaptic inhibition at the crayfish neuromuscular junction. J. Pfluegers Arch. 284: 66-80.

Dudel, J., and S. W. Kuffler (1961) Presynaptic inhibition at the crayfish neuromuscular junction. J. Physiol. (Lond.) 155: 543-562.

Fricke, R. A., G. D. Block, and D. Kennedy (1982) Inhibition of mechanosensory neurons in the crayfish II. Inhibition associated with proprioceptive feedback from locomotion. J. Comp. Physiol. 149: 251-262.

Fuchs, P. A., and P. A. Gelting (1980) Ionic basis of presynaptic inhibitory potentials at crayfish claw opener. J. Neurophysiol. 43: $1547-1557$.
Glantz, R. M. (1978) Crayfish antennal neuropil I. Reciprocal synaptic interactions and input-output characteristics of first-order interneurons. J. Neurophysiol. 41: 1297-1313.

Glantz, R. M., M. D. Kirk, and T. Viancour (1981) Interneurons of the crayfish brain: The relation between dendrite location and afferent input. J. Neurobiol. 12: 311-328.

Glantz, R. M., L. Wang-Bennett, and B. Waldrop (1985) Presynaptic inhibition in the crayfish brain. Inhibition of a central synapse and synaptic events in presynaptic terminals. J. Comp. Physiol. 156: 477487.

Graubard, K., J. A. Raper, and D. K. Hartline (1980) Graded synaptic transmission between spiking neurons. Proc. Natl. Acad. Sci. USA 77: 3733-3735.

Kandel, E. R., and L. Tauc (1965) Heterosynaptic facilitation in neurones of the abdominal ganglion of Aplysia depilans. J. Physiol. (Lond.) 181: $1-27$.

Kawai, A., and A. Niwa (1978) Hyperpolarization of the excitatory nerve terminals by inhibitory nerve stimulation in lobster. Brain Res. 137: $365-368$.

Kennedy, D., R. L. Calabrese, and J. J. Wine (1974) Presynaptic inhibition: Primary afferent depolarization in crayfish neurons. Science $186: 451-453$.

King, D. G. (1976) Organization of crustacean neuropil I. Patterns of synaptic connections in lobster stomatogastric ganglion. J. Neurocytol. 5: 207-237.

Kirk, M. D., and J. J. Wine (1984) Identified interneurons produce both primary afferent depolarization and presynaptic inhibition. Science 225: 854-856.

Klein, M., and E. R. Kandel (1980) Mechanism of calcium current modulation underlying presynaptic facilitation and behavioral sensitization in Aplysia. Proc. Natl. Acad. Sci. USA 77: 6912-6916.

Koketsu, K. (1956) Intracellular potential changes of primary afferent nerve fibers in spinal cords of cats. J. Neurophysiol. 19: 375-392.

Krasne, F. B., and J. S. Bryan (1973) Habituation: Regulation through presynaptic inhibition. Science 182: 590-592.

Nakajima, Y., A. D. Tisdale, and M. P. Henkart (1973) Presynaptic inhibition at inhibitory nerve terminals. A new synapse in the crayfish stretch receptor. Proc. Natl. Acad. Sci. USA 70: 2462-2466.

Shepherd, G. M. (1979) The Synaptic Organization of the Brain, p. 30, Oxford U.P., New York.

Stcwart, W. W. (1978) Functional connections between cells as rcvealed by dye-coupling with a highly fluorescent naphthalimide tracer. Cell 14: 741-759.

Uchizono, K. (1965) Characteristics of excitatory and inhibitory synapses in the central nervous system of the cat. Nature 207: 642-643.

Uchizono, K. (1967) Inhibitory synapses on the stretch receptor neurone of the crayfish. Nature 214: 833-834.

Wang-Bennett, L. T., and R. M. Glantz (1985) Presynaptic inhibition in the crayfish brain II. Morphology and ultrastructure of the terminal arborization. J. Comp. Physiol. 156: 605-617.

Wiersma, C. A. G., and B. M. H. Bush (1963) Functional neuronal connections between the thoracic and abdominal cords of the crayfish, Procambarus clarkii (Girard). J. Comp. Neurol. 121: 207-235.

Wiesma, C. A. G., and G. M. Hughes (1961) On the functional anatomy of neuronal units in the abdominal cord of the crayfish, Procambarus clarkii (Girard). J. Comp. Neurol. 116: 209-228.

Wiersma, C. A. G., and P. J. Mill (1965) “Descending” neuronal units in the commissure of the crayfish central nervous system; and their integration of visual, tactile, and proprioceptive stimuli. J. Comp. Neurol. 125: 67-94. 